\title{
When is radiofrequency ablation not indicated in head and neck squamous cell carcinoma management?
}

\author{
Leif J. J. Bäck • Antti A. Mäkitie • \\ Bert W. O'Malley Jr. • Randall P. Owen • \\ Alessandra Rinaldo $\cdot$ Carl E. Silver · Alfio Ferlito
}

Received: 12 March 2014 / Accepted: 12 May 2014 / Published online: 1 June 2014

(C) Springer-Verlag Berlin Heidelberg 2014

Regardless of the advances in treatment modalities used in head and neck squamous cell carcinoma (HNSCC), some patients present with untreatable disease associated with significant impairment of their quality of life, and will be offered palliative management. Life expectancy even with the use of extensive treatment approaches is less than a year for this group of patients [1]. One alternative for these patients is the use of the mini-invasive radiofrequency ablation (RFA) [2]. RFA technique involves direct placement of a wand that distributes alternating current into the tumor mass. Intratumoral temperatures of up to $100{ }^{\circ} \mathrm{C}$ are created

This paper was written by members and invitees of the International Head and Neck Scientific Group (www.IHNSG.com).

L. J. J. Bäck · A. A. Mäkitie

Department of Otorhinolaryngology-Head and Neck Surgery,

Helsinki University Central Hospital and University of Helsinki,

Helsinki, Finland

B. W. O’Malley Jr.

Department of Otorhinolaryngology-Head and Neck Surgery,

The University of Pennsylvania Health System, Philadelphia,

PA, USA

\section{R. P. Owen}

Division of Metabolic, Endocrine and Minimally Invasive Surgery, Department of Surgery, The Icahn School of Medicine at Mount Sinai, New York, NY, USA

A. Rinaldo $\cdot$ A. Ferlito $(\bowtie)$

University of Udine School of Medicine, Piazzale S. Maria della Misericordia, 33100 Udine, Italy

e-mail: a.ferlito@uniud.it

C. E. Silver

Departments of Surgery and Otolaryngology-Head and Neck Surgery, Albert Einstein College of Medicine, Montefiore

Medical Center, Bronx, NY, USA resulting in necrotic tumor and consequent volume shrinkage [2]. RFA has been used to treat unresectable malignant liver tumors with promising results [3], and some experience with its use in HNSCC for offering significant palliation has been acquired [4-6].

The volume of these procedures has arisen since 2004 [4] in several centers. However, no guidelines of RFA use in HNSCC exist and the related reports include encouraging results as well as treatment-related deaths, mainly caused by carotid artery rupture [4-6]. Thus, there is a clear call for widely accepted instructions including indications and contraindications for this treatment modality which would enable quality assurance guidelines. The present paper proposes device-, patient- and tumor-related contraindications for RFA in HNSCC.

\section{Device-related contraindications}

RFA with monopolar generators is contraindicated in patients with cardiac pacemakers or other nerve pacing devices as the generators can cause unwanted physiological effects. In addition, some treatment wands lack a complete protective shelf, which may cause problems to adjacent tissues along the ablation channel.

\section{Patient-related contraindications}

RFA is an attractive single modality choice due to its less invasive approach for this patient group with several comorbidities. However, difficult coagulopathies potentially increase the risk of adverse events. Treatment with acetylsalicylic acid is not a contraindication to RFA. It may be advisable to postpone the procedure in patients suffering 
from an active infection. Inability of patients to lie on their back on CT/MRI table if imaging guidance is needed has to be taken into account. Clear palliation targets must be considered and assessment with a planned follow-up protocol is encouraged. Non compliance with the follow-up protocol is a relative contraindication.

\section{Tumor-related contraindications}

At present, RFA should not be considered as an alternative to potentially curative salvage treatment options in HNSCC. Unless the patient has excessive disease affecting his physiology, we do not think that excessive tumor burden, metastases involving more than one organ or diffuse metastasis contraindicate RFA with palliative intent. In fact, every invasive modality with no evidence of true effect may be contraindicated and futile. This aspect must be discussed separately in each HNSCC case when considering RFA with palliative intent. RFA may still give added value in relief of symptoms in this subgroup of patients. Tumors positioned deeply close to essential structures, such as nerves or major blood vessels, with difficulties in creating a controlled lesion are challenging and associated with a high risk of failure. RFA should be avoided if the tumor mass borders the carotid artery. Further research may provide information as to the maximal tolerable dose in terms of delivered energy close to the carotid artery [2]. Most HNSCCs are easily accessible with this minimally invasive technique, but administration of RFA should be avoided for tumors with poor access even with CT/MRI guidance or for situations with other unfavorable geometry.

Failure rates are expected to be higher during the learning curve phase of all units which begin to offer RFA for HNSCC. The procedure should only be performed by surgeons with adequate training. Strong collaboration with clinicians, anesthesiologists and radiologist through a multidisciplinary meeting is mandatory to discuss each case and to decide whether the tumor meets the selection criteria. The Clavien Classification for surgical complications should be used to standardize morbidity reporting [7]. Strategies increasing the uniformity and completeness of RFA-induced tumor destruction are needed. Different interventions can potentially overcome many of the current restrictions posed when performing RFA as a single modality treatment.

Continuous assessment of the use of RFA is necessary to guarantee its potential role in the armamentarium of palliative treatment modalities for head and neck surgical oncologists. A major concern is the proximity of critical structures in HNSCC including major vessels, cranial nerves, airway, esophagus and pharynx. Without doubt, there is also potential to increase the distress of a patient with this modality. Accordingly, there is a need for discussion concerning the contraindications for and reporting of complications of RFA in HNSCC.

Conflict of interest The authors have no conflicts of interest or financial ties to disclose.

\section{References}

1. Vermorken JB, Stöhlmacher-Williams J, Davidenko I, Licitra L, Winquist E, Villanueva C, Foa P, Rottey S, Skladowski K, Tahara M, Pai VR, Faivre S, Blajman CR, Forastiere AA, Stein BN, Oliner KS, Pan Z, Bach BA, SPECTRUM investigators (2013) Cisplatin and fluorouracil with or without panitumumab in patients with recurrent or metastatic squamous-cell carcinoma of the head and neck (SPECTRUM): an open-label phase 3 randomised trial. Lancet Oncol 14:697-710

2. Owen RP, Lee JS, Silver CE, Bäck L, Beitler JJ, Rinaldo A, O'Malley BW Jr, Haigentz M Jr, Ferlito A (2014) Radiofrequency ablation in advanced head and neck cancer. Eur Arch Otorhinolaryngol 271:207-210

3. Hasegawa K, Aoki T, Ishizawa T, Kaneko J, Sakamoto Y, Sugawara Y, Kokudo N (2014) Comparison of the therapeutic outcomes between surgical resection and percutaneous ablation for small hepatocellular carcinoma. Ann Surg Oncol 21(Suppl 3):348-355

4. Owen RP, Silver CE, Ravikumar TS, Brook A, Bello J, Breining D (2004) Techniques for radiofrequency ablation of head and neck tumors. Arch Otolaryngol Head Neck Surg 130:52-56

5. Liukko T, Mäkitie AA, Markkola A, Ylikoski J, Bäck L (2006) Radiofrequency induced thermotherapy: an alternative palliative treatment modality in head and neck cancer. Eur Arch Otorhinolaryngol 263:532-536

6. Owen RP, Khan SA, Negassa A, Beitler JJ, Bello JA, Brook A, Farinhas J, Garg MK, Haigentz M Jr, Miller TS, Hsu MS, Ravikumar TS, Shifteh K, Smith RV, Silver CE (2011) Radiofrequency ablation of advanced head and neck cancer. Arch Otolaryngol Head Neck Surg 137:493-498

7. Dindo D, Demartines N, Clavien PA (2004) Classification of surgical complications: a new proposal with evaluation in a cohort of 6336 patients and results of a survey. Ann Surg 240:205-213 